\section{Crossed Cerebellar Diaschisis in a Child with Status Epilep- ticus}

Sir,

A 14-year boy was admitted to the Neurology Department for refractory status epilepticus, which had lasted for $>45$ minutes along with a documented fever of $102^{\circ} \mathrm{F}$. He was a known case of epilepsy for eight years and was on valproate, $500 \mathrm{mg}$, twice daily, but was never fit-free, with around one episode per month. During admission, he had a Glasgow coma score (GCS) of $8 / 15$, no signs of meningeal irritation, and planters were mute. Notably, he had multiple episodes of seizures during stay in the hospital. Baseline investigations were all within normal limits. EEG showed a slowing of left hemisphere activity with epileptiform discharges over left frontotemporal leads. MRI showed an extensive high signal change to the left cerebral hemisphere, right frontal lobe, and right cerebellar hemisphere in the superficial and deep white matter (Figure 1). Diffusion-weighted images (DWI) showed gyral restriction of diffusion in the left cerebral hemisphere, right frontal lobe, and right cerebellar hemisphere (Figure 2). The appearances in the left cerebral hemisphere and right cerebellar hemisphere are due to crossed cerebellardiaschisis (CCD).
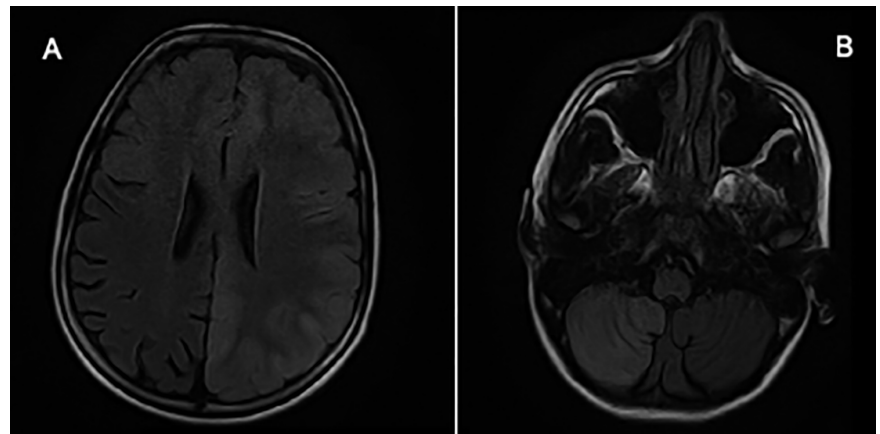

Figure 1: T2 and fluid attenuated inversion recovery (FLAIR) images. Axial FLAIR sequences showing hyperintense signals and cortical swelling in almost the entire left cerebral hemisphere, including basal ganglia, right frontal lobe (A), and right cerebellar hemisphere (B).

Figure 2: Diffusion-weighted Images.

Axial diffusion-weighted images showing restricted diffusion in entire left cerebral hemisphere $(A)$ and right cerebellar hemisphere $(B)$. Reciprocal changes were presentontheADCmap, confirming restricted diffusion.

CCD describes a depression of oxidative metabolism and blood flow in the cerebellum secondary to a supratentorial lesion in the contralateral cerebral hemisphere. ${ }^{1}$ This occurs in contrast to the usual ipsilateral thalamic signal that would normally be expected in such a case. ${ }^{2}$ The pathophysiology is not clear but appears to be caused by abnormal neuronal connection of the primary to the remote site. It has been reported mainly in stroke patients, patients with supratentorial tumors, migraines, and encephalitis. ${ }^{3}$ Although acute ischemic strokes in adults account for almost all of the reported cases of CCD, it has rarely been reported in a child with status epilepticus. This too, in which MRI of the brain with DWI was the tool for investigation as the usual investigation of diagnosis is a PETscan. ${ }^{4} \mathrm{CCD}$, secondary to status epilepticus, is extremely rare, and there is limited literature available on it. This is important because its findings can easily be confused with acute ischemic stroke and similar concurrent diseases. It is important to remember that peri-ictal MRI findings may result from the seizure activity rather than the cause; hence, this would avoid unnecessary and often risky diagnostic and therapeutic interventions. Thus, accurate diagnosis can also help localise the cause of the seizures and significantly influence surgical decisions.

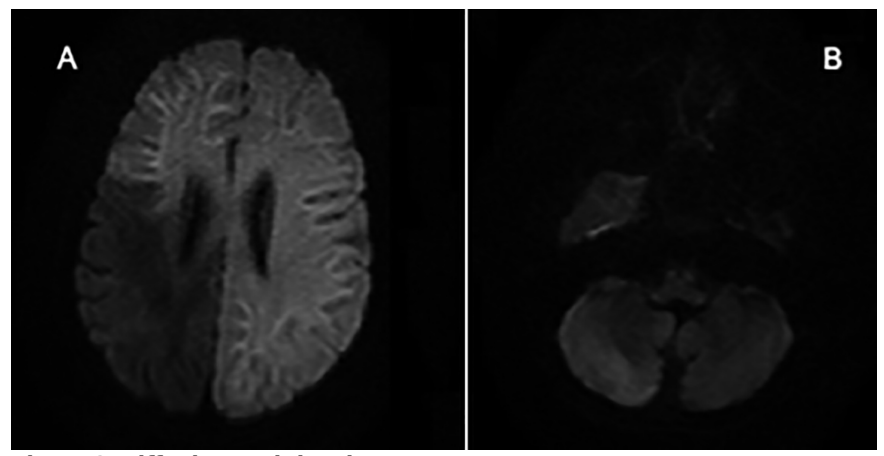

Figure 2: Diffusion-weighted Images.

Axial diffusion-weighted images showing restricted diffusion in entire left cerebral hemisphere (A) and right cerebellar hemisphere (B). Reciprocal changes were present on the ADC map, confirming restricted diffusion.

\section{PATIENT'S CONSENT:}

Informed consent regarding the publication of the case was obtained from the father of the patient.

\section{CONFLICT OF INTEREST:}

The authors declared no conflict of interest.

\section{AUTHORS' CONTRIBUTION:}

TD: Wrote the initial manuscript and revised it before submission.

MAUH: Ensured accuracy and integrity of the manuscript. $\mathrm{HI}, \mathrm{TF}, \mathrm{AK}$ : Interpreted radiographic findings and provided the relevant clinical correlation.

\section{REFERENCES}

1. Han S, Wang X, Xu K, Hu C. Crossed cerebellar diaschisis: Three case reports imaging using a tri-modality PET/CT-MR system. Medicine (Baltimore) 2016; 95(2):e2526-e. doi: 10.1097/MD.0000000000002526.

2. Zaidi SA, Haq MA, Bindman D, Mathur S. Crossed cerebellar diaschisis: A radiological finding in status epilepticus not to miss. BMJ Case Rep 2013; 2013:bcr2013200478. doi: 10.1136/bcr-2013-200478.

3. Katramados AM, Burdette D, Patel SC, Schultz LR, Gaddam S, Mitsias PD. Periictal diffusion abnormalities of the thalamus in partial status epilepticus. Epilepsia 2009; 50(2):265-75. doi: 10.1111/j.1528-1167.2008.01736.x. 
4. Sin DS, Kim MH, Park SA, Joo MC, Kim MS. Crossed cerebellar diaschisis: Risk factors and correlation to functional recovery in intracerebral hemorrhage. Ann Rehabil Med 2018; 42(1):8-17. doi: 10.5535/arm.2018. 42.1.8.

Talha Durrani', Mian Ayaz UI Haq ${ }^{2}$, Hira Ishaq ${ }^{2}$, Tanveer Fatima ${ }^{2}$ and Aamir Khan ${ }^{2}$

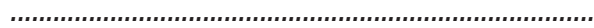

${ }^{1}$ Department of Surgery, Lady Reading Hospital, Peshawar, Pakistan
${ }^{2}$ Department of Neurology, Lady Reading Hospital, Peshawar, Pakistan

Correspondence to: Dr. Mian Ayaz Ul Haq, Department of Neurology, Lady Reading Hospital, Peshawar, Pakistan E-mail: drayazulhaq@gmail.com

Received: September 13, 2020; Revised: April 01, 2021; Accepted: April 18, 2021

DOI: https://doi.org/10.29271/jcpsp.2021.11.1387 